\section{Laser Doppler flowmetry as an assessment technique for localized scleroderma in children}

Localized scleroderma is a rare connective tissue disorder characterized by hardening and thickening of the skin due to increased collagen density. A quarter of patients affected also experience muscle and bone involvement. Methods to detect active lesions and monitor therapeutic efficacy are unreliable; although helpful to some degree, infrared thermography often yields falsepositive results. Laser Doppler flowmetry (LDF), a noninvasive method that measures cutaneous microcirculation, might be useful.

Weibel et al. compared the ability of LDF with that of infrared thermography to distinguish disease activity in 111 lesions from 41 children with localized scleroderma. All children underwent clinical examination, LDF, and thermography on two separate occasions. Seventy-five (34\%) lesions were clinically confirmed as being active and 147 (66\%) lesions as being inactive. A relative difference of $39 \%$ in blood flow and an absolute difference of $0.5^{\circ} \mathrm{C}$ in temperature were cutoff values for increased disease activity.

Median blood flow was increased by $89 \%$ in active lesions, compared with $11 \%$ in inactive lesions $(P<0.001)$. The corresponding median increases in temperature were $0.5^{\circ} \mathrm{C}$ and $0.3^{\circ} \mathrm{C}$, respectively $(P=0.024)$. Sensitivity values for LDF and thermography were $80 \%$ and $52 \%$, and specificity values were $77 \%$ and $58 \%$, respectively.

The authors conclude that LDF has diagnostic value because it can clearly and accurately distinguish active from inactive lesions. Further research into the ability of this method to monitor response to treatment is being performed.

Original article Weibel L et al. (2007) Laser Doppler flowmetry for assessing localized scleroderma in children. Arthritis Rheum 56: 3489-3495

\section{Validating MRI and the OMERACT RAMRIS for assessing joint damage in RA}

Documenting joint damage in rheumatoid arthritis (RA) is important for monitoring the course of the disease and for evaluating new treatments. MRI can be used for quantitative assessment of bone erosion volumes in the wrists and metacarpophalangeal (MCP) joints of RA patients. A semiquantitative scoring system, the Outcome Measures in Rheumatology Clinical Trials/Rheumatoid Arthritis Magnetic Resonance Imaging Score (OMERACT RAMRIS) has also been developed to assess bone erosions in these joints.

Neither scoring method has been sufficiently validated against any external reference, something that Møller Døhn et al. sought to rectify. They investigated agreement within and between methods when measuring erosion volumes of MCP joints on CT and MRI in 17 patients with RA and 4 healthy controls. They then compared the volumes with erosion scores for $\mathrm{CT}, \mathrm{MRI}$ and radiography.

$\mathrm{CT}, \mathrm{MRI}$ and radiography detected 77,62 and 12 erosions, respectively. The mean erosion volume seen on CT compared to MRI was very similar $\left(26 \mathrm{~mm}^{3}\right.$ vs $\left.30 \mathrm{~mm}^{3}\right)$. Total mean erosion volumes (per person, including controls) were $97 \mathrm{~mm}^{3}$ on CT and $90 \mathrm{~mm}^{3}$ on MRI. MRI erosion volumes correlated strongly with OMERACT RAMRIS erosion scores and with the Sharp/van der Heijde erosion score.

The authors say that their data support the reliability of MRI in estimating bone erosion size in RA, and demonstrate that the OMERACT RAMRIS erosion score is a valid measure of joint destruction.

Original article Møller Døhn U et al. (2007) Rheumatoid arthritis bone erosion volumes on CT and MRI: reliability and correlations with erosion scores on CT, MRI and radiography. Ann Rheum Dis 66: 1388-1392

\section{Increased fibrosis and number of myofibroblasts in the gastric wall of patients with SSc}

Organ failure, resulting from excessive fibrosis, is the main cause of morbidity and mortality in patients with systemic sclerosis (SSc). Dysfunction of the gastrointestinal (Gl) tract is a common feature of SSc. Manetti and co-workers have performed histologic analysis of gastric wall samples isolated from 14 patients with SSc to evaluate the presence of myofibroblasts, expression of the fibroblast-activating factors transforming growth factor $\beta$ (TGF- $\beta$ ), connective tissue growth factor (CTGF) and endothelin 1 (ET1), as well as the extent of collagen deposition.

Increased presence and patchy distribution of myofibroblasts were noted in the muscularis 